\title{
MiR-27a as a diagnostic biomarker and potential therapeutic target in systemic sclerosis
}

\section{Paria Bayati}

Iran University of Medical Sciences https://orcid.org/0000-0001-7371-9700

Mahsa Kalantari

IUMS: Iran University of Medical Sciences

Mohammad-Ali Assarehzadegan

IUMS: Iran University of Medical Sciences

Hadi Poormoghim

IUMS: Iran University of Medical Sciences

Nazanin Mojtabavi ( $\square$ Mojtabavi.n@iums.ac.ir)

IUMS: Iran University of Medical Sciences https://orcid.org/0000-0002-3539-9483

\section{Research}

Keywords: systemic sclerosis, SSc, scleroderma, miR-27a, miRNA, gene expression, microRNA

Posted Date: August 12th, 2021

DOl: https://doi.org/10.21203/rs.3.rs-770195/v1

License: (c) (1) This work is licensed under a Creative Commons Attribution 4.0 International License. Read Full License 


\section{Abstract \\ Background}

Systemic sclerosis (SSc) or scleroderma is a multiorgan rheumatoid disease characterized by skin tightening or organ dysfunction due to fibrosis, vascular damage, and autoimmunity. No specific cause has been discovered for this illness, and hence no effective treatment exists for it. On the other hand, due to the lack of diagnostic biomarkers capable of effectively and specifically differentiating the patients, early diagnosis has not been possible. Due to their potent regulatory roles in molecular pathways, microRNAs are among the novel candidates for the diagnosis and treatment of diseases like SSc. MiR-27a is a microRNA known for its role in the pathogenesis of fibrosis and cancer, both of which employ similar signaling pathways; hence we hypothesized that Mir-27a could be dysregulated in the blood of individuals affected by SSc and it might be useful in the diagnosis or treatment of this disease.

\section{Methods}

Blood was collected from 60 SSc patients (30 limited and 30 diffused) diagnosed by rheumatologist according to ACR/AULAR criteria; following RNA isolation and CDNA synthesis; real-time qPCR was performed on the samples using Taq-Man probes and data were analyzed by the $\triangle \Delta C T$ method. Also, potential targets of miR-27a were evaluated using bioinformatics.

\section{Results}

It was revealed that miR-27a was significantly down-regulated in SSc patients in comparison to the healthy individuals, but there was no difference in miR-27 expression between limited and diffused SSc patients. Besides, miR-27a was found to target several contributing factors to SSc.

\section{Conclusion}

It seems that miR-27a has a protective role in SSc, and its downregulation could result in the disease's onset. Based on bioinformatics analyses, it is speculated that miR-27a likely targets factors contributing to the pathogenesis of SSc, which are elevated upon the downregulation of miR-27a; hence, miR-27a mimics could be considered as potential therapeutic agents for the treatment of SSc in future studies. Since no difference was observed between limited and diffused patient groups, it is unlikely that this microRNA has a role in disease progression. According to ROC analysis of qPCR data, miR-27a could be employed as a valuable diagnostic biomarker for SSc.

\section{Background}

Systemic sclerosis (SSc) is an inflammatory disease of connective tissue with the hallmark characteristic of over-accumulation of the extracellular matrix, leading to fibrosis in the skin and different organs like lungs 
and kidneys; as a result, the affected individuals may experience vasculopathy and subsequently organ failure. The patients are placed into two distinct subcategories of limited and diffused, based on their symptoms and skin fibrosis pattern; with the limited form being the less severe, affecting only distal parts of the body and having less internal organs involvement, and the diffused form which affects the trunk and internal organs too (1). Patients affected with SSc experience a range of symptoms and complications; they are also prone to develop other autoimmune disorders like lupus and cancer (2). So far, there is no specific cure for this disease, mostly because there are many unknown aspects of this disease, and treatment approaches are symptom-oriented (3). Because of the wide range of symptoms that SSc bears, diagnostic approaches for this disease are also challenging and the most accepted criteria stated by the ACR/EULAR are also subject to change from time to time. Accordingly, there are different estimations concerning the prevalence and incidence of SSc; for instance, the prevalence of SSc in North America is reported as wide as 13.5 to 44.3 per 100,000 individuals (4). Since conventional treatment approaches are not helpful to cease SSc progression and efficiently ameliorate the symptoms, physicians and scientists consider exploring new approaches such as employing microRNAs in the treatment of SSc (5). Besides, finding an effective diagnostic biomarker that could facilitate diagnosing SSc at early onset, has been a major interest for researchers (6). Such a biomarker should be capable of identifying most of the patients or in other words, shows a high profile of sensitivity while also demonstrating a high profile of specificity as well. But currently, the majority of accepted diagnosing criteria for SSc are based on the disease complications rather than laboratory biomarkers; this results in the failure of the very early detection of SSc $(7,8)$. On the other hand, the available laboratory biomarkers such as auto-antibodies against topoisomerasel, centromere (SCL70) or RNA polymeraselll are not found in all SSc patients; besides, they are not much specific and are found in other rheumatoid diseases too. Furthermore, some newer biomarkers such as Lungen-6 (KL-6), surfactant protein-D (SP-D), and CCL18 are limited to the detection of complications such as interstitial lung disease (ILD) (9). As a result, exploring new approaches such as utilizing microRNAs which could detect the disease rather than the associated complications would be very promising for addressing the issues discussed so far (10).

MicroRNAs (miRNAs) are non-coding short sequences of ribonucleotides (about 22 nucleotides) that are capable of binding to other RNAs through their 3' UTR, which results in the blockade of protein synthesis from their targets or degradation of those targets (11). One of the most crucial features of miRNAs is their secretion from the host cell where they were transcribed and processed in the first place; hence they are present not only in the cells but unlike their mRNA targets, they can be found nearly in all of the body fluids including blood and even urine in a highly stable form since they are protected from RNases due to their interactions with proteins (12). This class of small RNAs is implicated in regulating different aspects of cellular physiology, including proliferation, apoptosis, and different signaling pathways, making them responsible for various abnormalities in cellular functions leading to diseases such as malignancies (1316). MiR-27, as its name implies is a microRNA, which is known for contributing to the pathogenesis of cancer, and also it is well known for being involved in adipogenesis $(17,18)$. One of the key players in fibrosis and associated disorders is TGF- $\beta$, which has a central role in the pathogenesis of SSc. Accordingly, a previous study has elucidated the role of miR-27 in a mouse model of pulmonary fibrosis as a negative regulator of TGF- $\beta R 1$ and SMAD2, which are crucial components of SSc pathogenesis too (19). It was also demonstrated that TGB- $\beta 1$ and smad3 are targeted by mir-27 in cervical cancer (20). Moreover, a different 
study on lung cancer has reported the concurrent upregulation of miR-27a and downregulation of two significant mediators of the TGF- $\beta$ signaling pathway, SMAD2, and SMAD4 (21). Among the potent regulators of the TGF- $\beta$ signaling pathway are PPAR- $\gamma$ and PTEN, both of which are reported to be targeted by miR-27a $(22,23)$. Besides, there are some studies regarding the effect of miR-27 on NF-kB which also acts downstream of the TGF- $\beta$ pathway $(24,25)$. The hallmark cytokine in fibrosis, interleukin- 6 and matrixmetalloproteinase 9 and 13, are also reported to be regulated by miR-27 (25). Moreover, it has been shown that miR-27a expression is also reduced in the lungs of IPF patients and bleomycin-induced lung fibrosis in mice (26).

There are common molecular pathways in cancer and fibrosis which is a hallmark of SSc, and since there is impaired adipogenesis in patients with SSc and mice models of this disease $(2,27-30)$; we aimed to evaluate the expression of miR-27a in the whole blood of SSc patients in order to find any deregulations and in case of obtaining a significant result, to test if it is possible to take advantage of miR-27 differential expression as a diagnostic tool.

\section{Materials And Methods}

Sixty female SSc patients (30 limited SSc and 30 diffused SSc) presenting to Firouzgar hospital, Tehran, Iran, were enrolled in this study. Male patients were excluded due to their low numbers. The diagnosis of the patients as well as categorizing them in limited and diffused subgroups was done based on ACR/EULAR criteria by an expert rheumatologist. All patients filled a consent form confirmed by the ethics committee of Iran University of medical sciences (ethic code: IR.IUMS.FMD.REC1396.30675). 20 consented healthy individuals matching the sex and age of the patients' group were also included as controls. Table 1 gives a concise description of the demographic features of the patients.

Table 1 demographic data and representative characteristics of the patients.

\begin{tabular}{|llllllllll|}
\hline number & $\begin{array}{l}\text { mean } \\
\text { age }\end{array}$ & sex & telangiectasia & $\begin{array}{l}\text { Renal } \\
\text { crisis }\end{array}$ & PAH $^{1}$ & ILD $^{2}$ & ARA $^{3}$ & $\begin{array}{l}\text { TOPO- } \\
\mathrm{I}^{4}\end{array}$ & ACA $^{5}$ \\
\hline 60 & 50.95 & female & $67.60 \%$ & $0 \%$ & $12.67 \%$ & $44.28 \%$ & $2.86 \%$ & $62.86 \%$ & $1.43 \%$ \\
\hline
\end{tabular}

${ }^{1}$ Pulmonary hypertension, ${ }^{2}$ Interstitial lung disease, ${ }^{3}$ Anti-RNA polymerase III, ${ }^{4}$ Anti-topoisomerase I, ${ }^{5}$ Anticentromere

Whole blood was collected from patients each day between 9 am to $12 \mathrm{pm}$ and was immediately added to RNAZOL-BD (MRC,USA) ( $0.5 \mathrm{ml}$ blood with $1 \mathrm{ml} \mathrm{RNAZOL),} \mathrm{and} \mathrm{total} \mathrm{RNA} \mathrm{was} \mathrm{isolated} \mathrm{according} \mathrm{to} \mathrm{the}$ manufacturer's protocol. After evaluating the RNA concentration and purity by NANODROP (Thermofisher); the RNA was converted into cDNA by TaqMan advanced microRNA synthesis kit (Ambion) according to the kit instructions. qPCR was carried out using TaqMan fast advanced microRNA fast master mix (Ambion) and TaqMan advanced microRNA assay (hsa-miR-27a-3p); thermal cycling was performed by RotorGene 6000(Qiagen) as recommended by the manufacturer protocol. hsa-miR-191a assay was employed for data normalization. Data were analyzed using $\triangle \triangle C T$ method and were compared using ANOVA by GraphPad PRISM software. P-values lower than 0.05 were considered statistically significant. 


\section{Bioinformatics analysis}

Using TargetScan, mirpath, mirpathDB, and KEGG pathway, we searched for putative targets of miR-27a which are known to be involved in fibrosis and hence SSc pathogenesis, specifically those contributing to the epithelial to the mesenchymal pathway (EMT).

\section{Results}

Realtime qPCR data are indicative of miR-27 presence in the whole blood. Comparison of RealTime qPCR data based on the expression of miR-27 between limited and diffused SSc patients and the healthy controls revealed a significant downregulation of miR-27 in both SSc groups compared with the healthy individuals; However, no difference was observed between the limited and diffused SSc patients (Fig. 1).

In order to evaluate the predictive value of miR-27 as a diagnostic marker for systemic sclerosis patients, receiver operative characteristic (ROC) analysis was performed and the resulting ROC curve showed a cutoff of $<9.975$ ( $\log 2$ of relative expression) and a good area under the curve (0.99) which is demonstrative of a good differentiating marker. The corresponding sensitivity and specificity of the selected cutoff were 0.96 and 0.95 respectively (Fig. 2).

TargetScan (http://www.targetscan.org) predicted 1613 genes to be possibly targeted by miR-27a, among those genes, we searched for the genes involved in the epithelial to mesenchymal transition (EMT) associated pathways, such as TGF- $\beta$ and PI3K/AKT/mTOR pathways; these genes were identified based on previous studies and data from KEGG as well as miRPathDB. It was found that miR-27a could target and regulate the expression of over 56 genes, known to be cardinals of EMT responsible for the transformation of fibroblasts into myofibroblasts; these cells overproduce high amounts of extracellular matrix components, including collagen. Table 1 lists the 56 genes predicted to be targeted by miR-27a, and Fig. 3 schematically illustrates these genes and their role in the development of fibrosis in SSc. 
Table 2

the genes involved in EMT/fibrosis pathway, which were predicted to be targeted and regulated by miR$27 a$.

\begin{tabular}{|c|c|c|}
\hline number & Gene symbol & Gene description \\
\hline 1 & IL6ST & interleukin 6 signal transducer (gp130, oncostatin M receptor) \\
\hline 2 & IGF1 & insulin-like growth factor 1 (somatomedin C) \\
\hline 3 & VEGFC & vascular endothelial growth factor $\mathrm{C}$ \\
\hline 4 & VEGFB & vascular endothelial growth factor $B$ \\
\hline 5 & FGF1 & fibroblast growth factor 1 \\
\hline 6 & FGF14 & fibroblast growth factor 14 \\
\hline 7 & FRS3 & fibroblast growth factor receptor substrate 3 \\
\hline 8 & HGF & hepatocyte growth factor \\
\hline 9 & PDK1 & pyruvate dehydrogenase kinase, isozyme 1 \\
\hline 10 & PDK4 & pyruvate dehydrogenase kinase, isozyme 4 \\
\hline 11 & F0X01 & forkhead box 01 \\
\hline 12 & F0X03 & forkhead box 03 \\
\hline 13 & BMP3 & bone morphogenetic protein 3 \\
\hline 14 & BMPR1A & bone morphogenetic protein receptor, type IA \\
\hline 15 & BMPR2 & bone morphogenetic protein receptor, type II \\
\hline 16 & WNT2B & wingless-type MMTV integration site family, member 2B \\
\hline 17 & WNT3A & wingless-type MMTV integration site family, member $3 \mathrm{~A}$ \\
\hline 18 & TGIF2 & TGFB-induced factor homeobox 2 \\
\hline 19 & GSK3B & glycogen synthase kinase 3 beta \\
\hline 20 & F0X01 & forkhead box 01 \\
\hline 21 & F0X03 & forkhead box 03 \\
\hline 22 & PPARG & peroxisome proliferator-activated receptor gamma \\
\hline 23 & Table 2 & TGF-beta activated kinase 1/MAP3K7 binding protein 2 \\
\hline 24 & Table 3 & TGF-beta activated kinase 1/MAP3K7 binding protein 3 \\
\hline 25 & TGFBR1 & transforming growth factor, beta receptor 1 \\
\hline 26 & TGFBR2 & transforming growth factor, beta receptor II \\
\hline 27 & TGFBR3 & transforming growth factor, beta receptor III \\
\hline
\end{tabular}




\begin{tabular}{|c|c|c|}
\hline number & Gene symbol & Gene description \\
\hline 28 & SMAD2 & SMAD family member 2 \\
\hline 29 & SMAD5 & SMAD family member 5 \\
\hline 30 & SMAD9 & SMAD family member 9 \\
\hline 31 & ITGA1 & integrin, alpha 1 \\
\hline 32 & ITGA2 & integrin, alpha 2 (CD49B, alpha 2 subunit of VLA-2 receptor) \\
\hline 33 & ITGA5 & integrin, alpha 5 (fibronectin receptor, alpha polypeptide) \\
\hline 34 & ITGA8 & integrin, alpha 8 \\
\hline 35 & ITGB8 & integrin, beta 8 \\
\hline 36 & $\mathrm{SHH}$ & sonic hedgehog \\
\hline 37 & FZD3 & frizzled family receptor 3 \\
\hline 38 & FZD4 & frizzled family receptor 4 \\
\hline 39 & GAREM & GRB2 associated regulator of MAPK1 \\
\hline 40 & MAPK10 & mitogen-activated protein kinase 10 (JNK) \\
\hline 41 & MAPK14 & mitogen-activated protein kinase 14 (p38) \\
\hline 42 & MAPKAPK3 & mitogen-activated protein kinase-activated protein kinase 3 \\
\hline 43 & MAPK8IP3 & mitogen-activated protein kinase 8 interacting protein 3 \\
\hline 44 & MMP13 & matrix metallopeptidase 13 \\
\hline 45 & MMP16 & matrix metallopeptidase 16 \\
\hline 46 & SNAI1 & snail family zinc finger 1 \\
\hline 47 & FN1 & fibronectin 1 \\
\hline 48 & ELFN2 & extracellular leucine-rich repeat and fibronectin type III domain containing 2 \\
\hline 49 & FLRT2 & fibronectin leucine rich transmembrane protein 2 \\
\hline 50 & FLRT3 & fibronectin leucine rich transmembrane protein 3 \\
\hline 51 & FNDC3A & fibronectin type III domain containing $3 \mathrm{~A}$ \\
\hline 52 & FNDC4 & fibronectin type III domain containing 4 \\
\hline 53 & COL5A1 & collagen, type $\mathrm{V}$, alpha 1 \\
\hline 54 & COL11A2 & collagen, type XI, alpha 2 \\
\hline 55 & HYOU1 & hypoxia up-regulated 1 \\
\hline 56 & SIX1 & SIX homeobox 1 \\
\hline
\end{tabular}




\section{Discussion}

Fibrosis-associated disorders like systemic sclerosis (SSc) annually cause the morbidity and mortality of thousands of people around the world; yet there is a lot to be understood to unravel the exact mechanisms involved and hence no curative treatment plan exists for diseases like SSc. The broad range of symptoms and complications and also disease severity are among the main causes that this illness is not fully uncovered. Of the new research targets are microRNAs, which are implicated in all aspects of cell biology, and their roles in different diseases are of significant interest to scientists. Based on the previous studies regarding the role of miR-27 in cancer and fibrosis, which share many similar pathologic aspects especially regarding the EMT, and since this microRNA is implicated in the regulation of the TGF- $\beta$ signaling pathway as a cardinal regulator of fibrosis, we sought to assess the expression of miR-27 in whole blood of the SSc patients to evaluate its usefulness in the early diagnosis of SSc patients. Also, we aimed to evaluate its capability for distinguishing limited and diffused subsets of the disease, if applicable. The role of miRNAs in fibrosis-associated disorders has been widely studied, and it has been shown that miRNAs can target different aspects of fibrotic procedures (31).

In the current study, the expression of miR-27 in the whole blood of SSc patients was investigated by taking advantage of Real-time qPCR; the statistical analyses demonstrated a significant downregulation of miR-27 in the SSc patients compared with the healthy controls. According to the ROC analysis, a highly specific and sensitive cutoff was assigned to the qPCR data, suggesting an excellent diagnostic value of miR-27a for diagnosing SSc. We had presumed that the relative expression of miR-27 in diffused SSc patients is different from the limited ones, but the results were similar in both groups; hence we couldn't propose miR-27 as a differentiating laboratory tool for precise subcategorization of SSc patients. The lack of difference between the two subsets of SSc patients may indicate miR-27 contributes to disease onset, not in disease progression, although more investigations are required to make a decisive conclusion. There were no recently diagnosed patients in our study, and the majority had an average five years history of being diagnosed with SSc. Due to the challenges regarding the diagnosis of these patients in the early stages, we were unable to find a minimum number of newly diagnosed patients required for obtaining statistically significant results. We also conducted statistical analysis to find correlations between miR-27a expression and patient symptoms like interstitial lung disease (ILD), telangiectasia, renal crisis, pulmonary arterial hypertension, etc. (data not shown). We couldn't find any significant correlation between miR-27a expression and patient symptoms, although a weak non-significant $(p$ value $=0.09)$ negative correlation was observed with miR-27a relative expression and ILD, which might be significant if the number of patients was higher. This also may confirm the role of miR-27a as a basic causal factor rather than a situation-dependent factor contributing to a specific complication of the disease.

As illustrated in Fig. 3, miR-27a was predicted to play an essential role in the fibrosis process. This microRNA targets various gene transcripts involved in the differentiation of fibroblasts into myofibroblasts during the process of EMT, which serves as the basis for the development of fibrosis-associated complications, including skin thickening, vascular problems, and organ fibrosis. In this regard, we found different studies, which confirm the inhibitory effect of miR-27a on the predicted genes. It was shown that miR-27a suppresses inflammation by targeting IL-6 and MAP kinases like P38 and JNK (32). Besides, miR-27a was found to 
inhibit the expression of SMAD signaling mediators that act downstream of the TGF- $\beta$ pathway (33). Another inhibitory effect of miR-27a on the TGF- $\beta$ signaling pathway is mediated via direct targeting of TGF- $\beta$ RI transcript (20). Also, major growth factors capable of contributing to fibrosis pathogenesis were found to be downregulated by miR-27a, including IGF-1 and VEGF $(34,35)$. All of the aforementioned studies are in line with our results which strongly demonstrate the protective effect of miR-27a against fibrosis and hence SSc. As depicted in Fig. 3, miR-27a also targets and regulates MMP13 which is a matrix metalloproteinase involved in the remodeling of extracellular matrix during fibrotic changes (36).

MiR-27a has been studied widely in cancer and metabolism(37), but to our knowledge, this is the first study considering the role of miR-27a in systemic sclerosis, although it needs further functional analyses in order to give a comprehensive and conclusive outcome. The remarkable finding of our study is the downregulation of miR-27a in the whole blood of patients affected with systemic sclerosis (either limited or diffused) in comparison to healthy individuals. Thus, we could propose a role for miR-27a in SSc; it seems likely that miR-27 negatively affects molecular pathways involved in fibrogenesis, such as the TGF- $\beta$ signaling pathway, and as a result, it could be considered as an anti-fibrotic microRNA. In support of our theory Cui $\mathrm{H}$, et al. investigated the therapeutic effect of miR-27a on pulmonary fibrosis by demonstrating the inhibitory effect of miR-27a on SMAD2 and SMAD4, which are the major mediators of the TGF- $\beta$ signaling pathway; They have shown that $\alpha$-smooth muscle actin is directly regulated by miR-27a as well, which is the characteristic feature of myofibroblast differentiation (38). Also, there are other shreds of evidence regarding the relationship between miR-27a and the TGF- $\beta$ signaling pathway in other circumstances. Consistent with our theory, Fang Fang, et al. have shown the ability of miR-27a in inhibition of the TGF- $\beta$ signaling pathway through suppression of TGF- $\beta R$ I, Smad2, and Smad4 expression; as well as reduced phosphorylation of Smad3 in cervical adenocarcinoma; cell proliferation, migration, and invasion which employ the same mechanism of EMT in fibrosis, were also attenuated (20). Dong-Kyu Chae et al. have shown the negative regulation of SMAD2, and SMAD4 by miR-27a in lung cancer cell lines (39). Consistently, Qi Xu, et al. have demonstrated that miR-27a suppresses TGF- $\beta$ induced expression of SMAD4 in human lymphatic endothelial cells, which supports our hypothesis on the role of miR-27a as a likely anti-fibrotic factor (40). it is noteworthy to mention that in a model of myocardial ischemia, Zhang Xl, et al. have shown the downregulation of TGFBRI along with the reduction of IL-6, TNF- $a$, and p-NFKB by miR-27a (41); all of which are major inflammatory factors contributing to fibrosis pathogenesis. On the contrary, Hui Zhang, et al. have investigated the role of miR-27a in hepatic stellate cell (HSC) activation and fibrosis and concluded that miR-27a increases in response to TGF- $\beta$ and contributes to HSC activation and fibrosis; they have demonstrated that the inhibition of miR-27a leads to the suppression of PPARy, a-SMA and collagen too (42).Quin Lin, Et al. and Sun Young Jang, et al. in two different but similar studies on adipogenesis, have shown that the overexpression of miR-27a results in the downregulation of PPARY, which is known as an inhibitor of TGF- $\beta$ non-canonical pathway $(23,43)$. In this regard, Deng K, et al. have shown the negative regulation of PPARY by miR-27a, too (44). Besides, in a study conducted by Ji-Hoon Cho, et al. with a systems biology approach, it was found that miR-27a along with miR-23a and miR24 is upregulated in response to TGF- $\beta$ by ZEB-1 transcription factor which is a major participant in EMT (35). It could be suggested that miR-27a elevation in these instances may take place upon over-activation of the TGF- $\beta$ signaling pathway as a negative feedback mechanism to attenuate its physiological consequences. The other regulator of the TGF- $\beta$ pathway, PTEN, has also been considered as a target of miR-27a; In an 
interesting study, though on diabetic mice models, Aiqing Zhang, et al. have shown the ability of miR-23amir-27a cluster in downregulation of Fox01 and PTEN; they have also demonstrated that the expression of TGF- $\beta$ and pSMAD3/4 are suppressed by miR-27a thereby resulting in the attenuation of renal fibrosis (45). Collectively we believe that miR-27a has a regulatory role regarding the signaling pathways involved in fibrosis and therefore SSc, and the majority of data support the idea that miR-27a has a protective effect against SSc. Also, it is likely that a genetic deficit or any contributing factor for SSc causes the downregulation of miR-27a thereby leading to the aberrant activation of TGF- $\beta$ and other signaling pathways contributing to SSc pathogenesis.

\section{Conclusion}

Based on the statistical analyses, miR-27a could serve as a reliable diagnostic marker for SSc, and given its proposed role in regulating TGF- $\beta$ and other contributing pathways to SSc, it could be considered as a treatment option both for SSc and its related disorders and complications, which indeed necessitates further investigations.

\section{Abbreviations}

ACA: Anti-centromere

ACR/AULAR: The European League Against Rheumatism/American College of Rheumatology

ARA: Anti-RNA polymerase III

BMP3: bone morphogenetic protein 3

BMPR1A: bone morphogenetic protein receptor, type IA

BMPR2: bone morphogenetic protein receptor, type II

CCL18: C-C Motif Chemokine Ligand 18

cDNA: Complementary DNA

COL11A2: collagen, type XI, alpha 2

COL5A1: collagen, type $\mathrm{V}$, alpha 1

ELFN2: extracellular leucine-rich repeat and fibronectin type III domain containing 2

EMT: epithelial to mesenchymal transition (EMT(

FGF1: fibroblast growth factor 1

FGF14: fibroblast growth factor 14 
FLRT2: fibronectin leucine rich transmembrane protein 2

FLRT3: fibronectin leucine rich transmembrane protein 3

FN1: fibronectin 1

FNDC3A: fibronectin type III domain containing 3A

FNDC4: fibronectin type III domain containing 4

F0X01: forkhead box 01

F0X01: forkhead box 01

F0X03: forkhead box 03

FOX03: forkhead box 03

FRS3: fibroblast growth factor receptor substrate 3

FZD3: frizzled family receptor 3

FZD4: frizzled family receptor 4

GAREM: GRB2 associated regulator of MAPK1

GSK3B: glycogen synthase kinase 3 beta

HGF: hepatocyte growth factor

HSC: hepatic stellate cell

HYOU1: hypoxia up-regulated 1

IGF1: insulin-like growth factor 1 (somatomedin C(

IL6ST: interleukin 6 signal transducer (gp130, oncostatin M receptor(

ILD: interstitial lung disease

IPF: Idiopathic pulmonary fibrosis

ITGA1: integrin, alpha 1

ITGA2: integrin, alpha 2

ITGA5: integrin, alpha 5

ITGA8: integrin, alpha 8 
ITGB8: integrin, beta 8

KL-6: Lungen-6

MAPK10: mitogen-activated protein kinase 10 (JNK(

MAPK14: mitogen-activated protein kinase 14 (p38(

MAPK8IP3: mitogen-activated protein kinase 8 interacting protein 3

MAPKAPK3: mitogen-activated protein kinase-activated protein kinase 3

miR: microRNA

miRNAs: microRNAs

MMP13: matrix metallopeptidase 13

MMP16: matrix metallopeptidase 16

NF-KB: nuclear factor kappa-light-chain-enhancer of activated B cells

PAH: Pulmonary hypertension

PDK1: pyruvate dehydrogenase kinase, isozyme 1

PDK4: pyruvate dehydrogenase kinase, isozyme 4

PPAR-ү: Peroxisome proliferator-activated receptor

PTEN: Phosphatase and tensin homolog

Quantitative Polymerase Chain Reaction: qPCR

ROC: Receiver operating characteristic curve

SHH: sonic hedgehog

SIX1: SIX homeobox 1

SMAD2: SMAD family member 2

SMAD5: SMAD family member 5

SMAD9: SMAD family member 9

SNAl1: snail family zinc finger 1

SP-D: surfactant protein-D 
SSc: Systemic sclerosis (SSc(

TAB2: TGF-beta activated kinase 1/MAP3K7 binding protein 2

TAB3: TGF-beta activated kinase 1/MAP3K7 binding protein 3

TGFBR1: transforming growth factor, beta receptor 1

TGFBR2: transforming growth factor, beta receptor II

TGFBR3: transforming growth factor, beta receptor III

TGF- $\beta$ : transforming growth factor beta

TGF- $\beta R 1$ : transforming growth factor beta receptor1

TGIF2: TGFB-induced factor homeobox 2

TOPO-I: Anti-topoisomerase I

3'UTR: 3' untranslated region

VEGFB: vascular endothelial growth factor B

VEGFC: vascular endothelial growth factor C

WNT2B: wingless-type MMTV integration site family, member 2B

WNT3A: wingless-type MMTV integration site family, member 3A

\section{Declarations}

Ethics approval and consent to participate:

All patients filled a consent form confirmed by the ethics committee of Iran University of medical sciences (ethic code: IR.IUMS.FMD.REC1396.30675).

Consent for publication:

Not applicable.

Availability of data and materials:

The datasets used and/or analyzed during the current study are available from the corresponding author on reasonable request.

Competing interests:

The authors declare that they have no competing interests. 
Funding:

This study was supported by a grant from the Iran University of Medical Sciences.

Grant Number:96013030675

Authors' contributions

PB: sample preparations, statistical/bioinformatics analyses, and preparing the manuscript. MK was involved in the study design and collection of blood samples. MAA helped with the study design and conduction as well as editing the manuscript. HP: patients gathering and diagnosis and also edited the manuscript. NM has designed the study and provided the study materials; also she has guided all the study procedures from the first idea to the preparation of the manuscript.

All authors have read and approved the final manuscript.

Acknowledgments

$\mathrm{N} / \mathrm{A}$

\section{References}

1. Sobolewski P, Maślińska M, Wieczorek M, Łagun Z, Malewska A, Roszkiewicz M, et al. Systemic sclerosis - multidisciplinary disease: clinical features and treatment. Reumatologia. 2019;57(4):221-33.

2. Morrisroe K, Hansen D, Huq M, Stevens W, Sahhar J, Ngian GS, et al. Incidence, risk factors and outcomes of cancer in systemic sclerosis. Arthritis Care Res (Hoboken). 2019.

3. Fernandez-Codina A, Walker KM, Pope JE. Treatment Algorithms for Systemic Sclerosis According to Experts. Arthritis Rheumatol. 2018;70(11):1820-8.

4. Bergamasco A, Hartmann N, Wallace L, Verpillat P. Epidemiology of systemic sclerosis and systemic sclerosis-associated interstitial lung disease. Clinical epidemiology. 2019;11:257-73.

5. Henry TW, Mendoza FA, Jimenez SA. Role of microRNA in the pathogenesis of systemic sclerosis tissue fibrosis and vasculopathy. Autoimmun rev. 2019;18(11):102396.

6. Zhang L, Wu H, Zhao M, Lu Q. Meta-analysis of differentially expressed microRNAs in systemic sclerosis. International Journal of Rheumatic Diseases. 2020;23(10):1297-304.

7. van den Hoogen F, Khanna D, Fransen J, Johnson SR, Baron M, Tyndall A, et al. 2013 classification criteria for systemic sclerosis: an American College of Rheumatology/European League against Rheumatism collaborative initiative. Arthritis Rheum. 2013;65(11):2737-47.

8. Lepri G, Randone SB, Bruni C, Matucci-Cerinic M, Guiducci S. Very early and early diagnosis of systemic sclerosis. Novel Insights into Systemic Sclerosis Management. Future Medicine Ltd; 2013. pp. 52-60.

9. Utsunomiya A, Oyama N, Hasegawa M. Potential Biomarkers in Systemic Sclerosis: A Literature Review and Update. Journal of clinical medicine. 2020;9(11). 
10. Condrat CE, Thompson DC, Barbu MG, Bugnar OL, Boboc A, Cretoiu D, et al. miRNAs as Biomarkers in Disease: Latest Findings Regarding Their Role in Diagnosis and Prognosis. Cells. 2020;9(2):276.

11. Wahid F, Shehzad A, Khan T, Kim YY. MicroRNAs. Synthesis, mechanism, function, and recent clinical trials. Biochimica et Biophysica Acta (BBA) -. Molecular Cell Research. 2010;1803(11):1231-43.

12. O'Brien J, Hayder H, Zayed Y, Peng C. Overview of MicroRNA Biogenesis, Mechanisms of Actions, and Circulation. Frontiers in Endocrinology. 2018;9:402.

13. Peng Y, Croce CM. The role of MicroRNAs in human cancer. Signal Transduction Targeted Therapy. 2016;1(1):15004.

14. Papagiannakopoulos T, Kosik KS. MicroRNAs: regulators of oncogenesis and stemness. BMC Med. 2008;6(1):15.

15. Brothers JF, Hijazi K, Mascaux C, El-Zein RA, Spitz MR, Spira A. Bridging the clinical gaps: genetic, epigenetic and transcriptomic biomarkers for the early detection of lung cancer in the post-National Lung Screening Trial era. BMC Med. 2013;11(1):168.

16. To KKW. MicroRNA: a prognostic biomarker and a possible druggable target for circumventing multidrug resistance in cancer chemotherapy. J Biomed Sci. 2013;20(1):99.

17. Li X, Xu M, Ding L, Tang J. MiR-27a: A Novel Biomarker and Potential Therapeutic Target in Tumors. J Cancer. 2019;10(12):2836-48.

18. Kulyté A, Kwok KHM, de Hoon M, Carninci P, Hayashizaki Y, Arner P, et al. MicroRNA-27a/b-3p and PPARG regulate SCAMP3 through a feed-forward loop during adipogenesis. Sci Rep. 2019;9(1):13891.

19. Zeng X, Huang C, Senavirathna L, Wang P, Liu L. miR-27b inhibits fibroblast activation via targeting TGF $\beta$ signaling pathway. BMC Cell Biol. 2017;18(1):9-.

20. Fang F, Huang B, Sun S, Xiao M, Guo J, Yi X, et al. miR-27a inhibits cervical adenocarcinoma progression by downregulating the TGF- $\beta$ RI signaling pathway. Cell Death Dis. 2018;9(3):395.

21. Chae D-K, Ban E, Yoo YS, Kim EE, Baik J-H, Song EJ. MIR-27a regulates the TGF- $\beta$ signaling pathway by targeting SMAD2 and SMAD4 in lung cancer. Molecular carcinogenesis. 2017;56(8):1992-8.

22. Wang B, Zhang C, Zhang A, Cai H, Price SR, Wang XH. MicroRNA-23a and MicroRNA-27a Mimic Exercise by Ameliorating CKD-Induced Muscle Atrophy. J Am Soc Nephrol. 2017;28(9):2631.

23. Lin Q, Gao Z, Alarcon RM, Ye J, Yun Z. A role of miR-27 in the regulation of adipogenesis. FEBS J. 2009;276(8):2348-58.

24. Freudlsperger C, Bian Y, Contag Wise S, Burnett J, Coupar J, Yang X, et al. TGF-beta and NF-kappaB signal pathway cross-talk is mediated through TAK1 and SMAD7 in a subset of head and neck cancers. Oncogene. 2013;32(12):1549-59.

25. Zhou B, Li H, Shi J. miR27 inhibits the NF-kappaB signaling pathway by targeting leptin in osteoarthritic chondrocytes. Int J Mol Med. 2017;40(2):523-30.

26. Cui H, Banerjee S, Xie N, Ge J, Liu RM, Matalon S, et al. MicroRNA-27a-3p Is a Negative Regulator of Lung Fibrosis by Targeting Myofibroblast Differentiation. Am J Respir Cell Mol Biol. 2016;54(6):843-52.

27. Marasini B, Conciato L, Belloli L, Massarotti M. Systemic sclerosis and cancer. Int J Immunopathol Pharmacol. 2009;22(3):573-8. 
28. Korman B, Marangoni RG, Lord G, Olefsky J, Tourtellotte W, Varga J. Adipocyte-specific Repression of PPAR-gamma by NCoR Contributes to Scleroderma Skin Fibrosis. Arthritis research therapy. 2018;20(1):145-.

29. Varga J, Marangoni RG. Dermal white adipose tissue implicated in SSc pathogenesis. Nat Rev Rheumatol. 2017;13:71.

30. Ohgo S, Hasegawa S, Hasebe Y, Mizutani H, Nakata S, Akamatsu H. Bleomycin inhibits adipogenesis and accelerates fibrosis in the subcutaneous adipose layer through TGF-beta1. Exp Dermatol. 2013;22(11):769-71.

31. Vettori S, Gay S, Distler O. Role of MicroRNAs in Fibrosis. Open Rheumatol J. 2012;6:130-9.

32. Min S, Li L, Zhang M, Zhang Y, Liang X, Xie Y, et al. TGF- $\beta$-associated miR-27a inhibits dendritic cellmediated differentiation of Th1 and Th17 cells by TAB3, p38 MAPK, MAP2K4 and MAP2K7. Genes Immunity. 2012;13(8):621-31.

33. Chae DK, Ban E, Yoo YS, Kim EE, Baik JH, Song EJ. MIR-27a regulates the TGF- $\beta$ signaling pathway by targeting SMAD2 and SMAD4 in lung cancer. Molecular carcinogenesis. 2017;56(8):1992-8.

34. Chen Y, Zhang X, An Y, Liu B, Lu M. LncRNA HCP5 promotes cell proliferation and inhibits apoptosis via miR-27a-3p/IGF-1 axis in human granulosa-like tumor cell line KGN. Mol Cell Endocrinol. 2020;503:110697.

35. Zhang W, Chen L, Geng J, Liu L, Xu L. $\beta$-elemene inhibits oxygen-induced retinal neovascularization via promoting miR-27a and reducing VEGF expression. Mol Med Rep. 2019;19(3):2307-16.

36. Elahe I, Fateme V, Paria J, Pendar S, Mohamad Ali A, Hadi P, et al. Effect of Progesterone on Expression of MMP7 and MMP13 in Lungs of Female Mice. Iranian Journal of Allergy, Asthma and Immunology. 2018;17(5).

37. Zhang J, Cao Z, Yang G, You L, Zhang T, Zhao Y. MicroRNA-27a (miR-27a) in Solid Tumors: A Review Based on Mechanisms and Clinical Observations. Frontiers in Oncology. 2019;9(893).

38. Cui H, Banerjee S, Xie N, Ge J, Liu R-M, Matalon S, et al. MicroRNA-27a-3p Is a Negative Regulator of Lung Fibrosis by Targeting Myofibroblast Differentiation. Am J Respir Cell Mol Biol. 2016;54(6):843-52.

39. Chae DK, Ban E, Yoo YS, Kim EE, Baik JH, Song EJ. MIR-27a regulates the TGF-beta signaling pathway by targeting SMAD2 and SMAD4 in lung cancer. Molecular carcinogenesis. 2017;56(8):1992-8.

40. Xu Q, Tong J-L, Zhang C-P, Xiao Q, Lin X-L, Xiao X-Y. miR-27a induced by colon cancer cells in HLECs promotes lymphangiogenesis by targeting SMAD4. PloS one. 2017;12(10):e0186718-e.

41. Zhang X-L, An B-F, Zhang G-C. MiR-27 alleviates myocardial cell damage induced by hypoxia/reoxygenation via targeting TGFBR1 and inhibiting NF-kB pathway. The Kaohsiung Journal of Medical Sciences. 2019;35(10):607-14.

42. Zhang H, Yan X-L, Guo X-X, Shi M-J, Lu Y-Y, Zhou Q-M, et al. MiR-27a as a predictor for the activation of hepatic stellate cells and hepatitis B virus-induced liver cirrhosis. Oncotarget; Vol 9, No 1. 2017.

43. Jang SY, Chae MK, Lee JH, Lee EJ, Yoon JS. MicroRNA-27 inhibits adipogenic differentiation in orbital fibroblasts from patients with Graves' orbitopathy. PloS one. 2019;14(8):e0221077-e.

44. Deng K, Ren C, Fan Y, Liu Z, Zhang G, Zhang Y, et al. miR-27a is an important adipogenesis regulator associated with differential lipid accumulation between intramuscular and subcutaneous adipose 
tissues of sheep. Domest Anim Endocrinol. 2020;71:106393.

45. Zhang A, Li M, Wang B, Klein JD, Price SR, Wang XH. miRNA-23a/27a attenuates muscle atrophy and renal fibrosis through muscle-kidney crosstalk. Journal of cachexia sarcopenia muscle. 2018;9(4):75570.

Figures

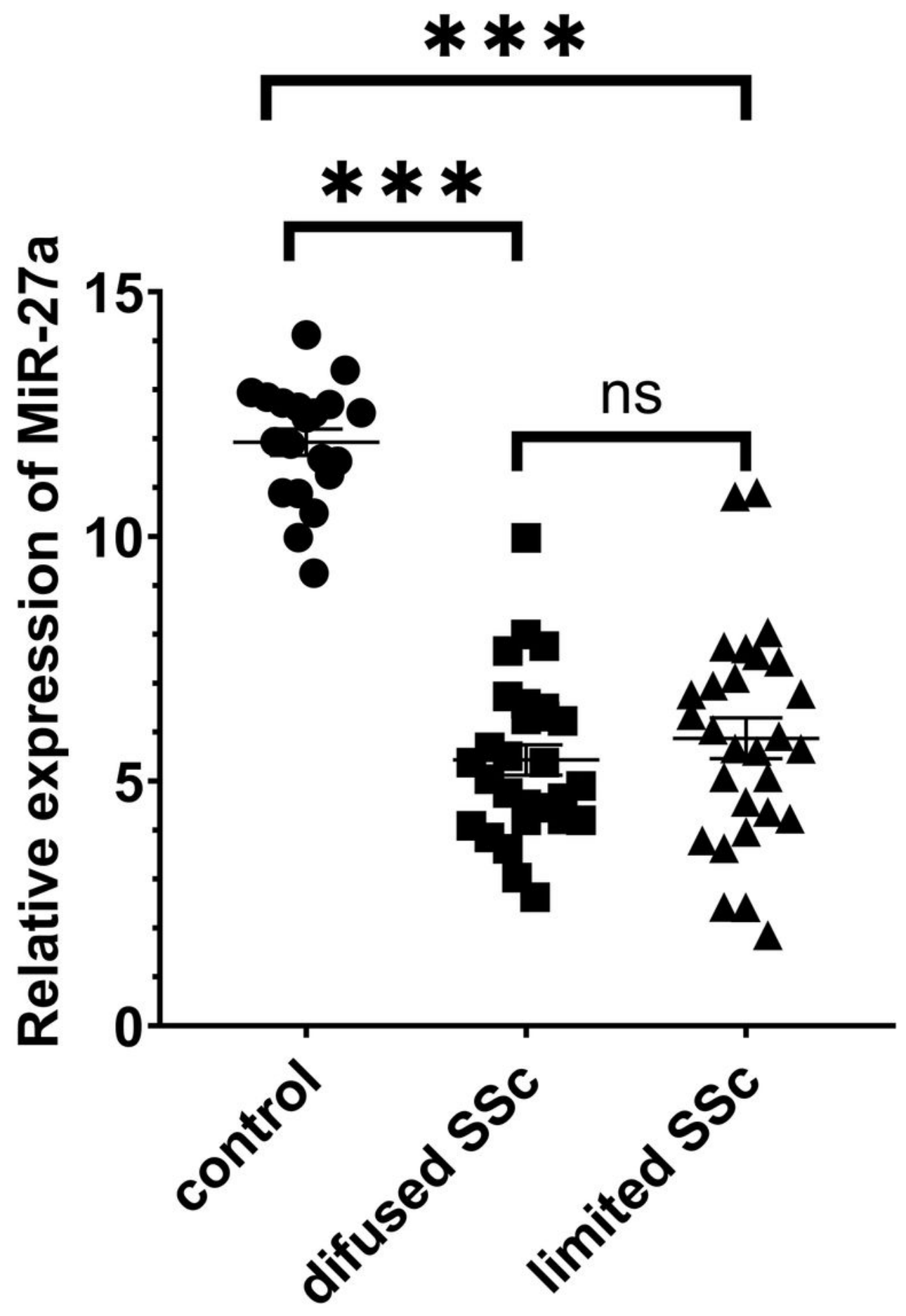

Figure 1 
Relative expression of miR-27 in healthy control and patient with diffused or limited systemic sclerosis. Data are shown as mean \pm SEM. The expression of miR-27a is downregulated in SSc patients compared with the controls. P-value $=0.0001$. There's no significant difference in the expression of miR-27a between diffused and limited SSc patients.

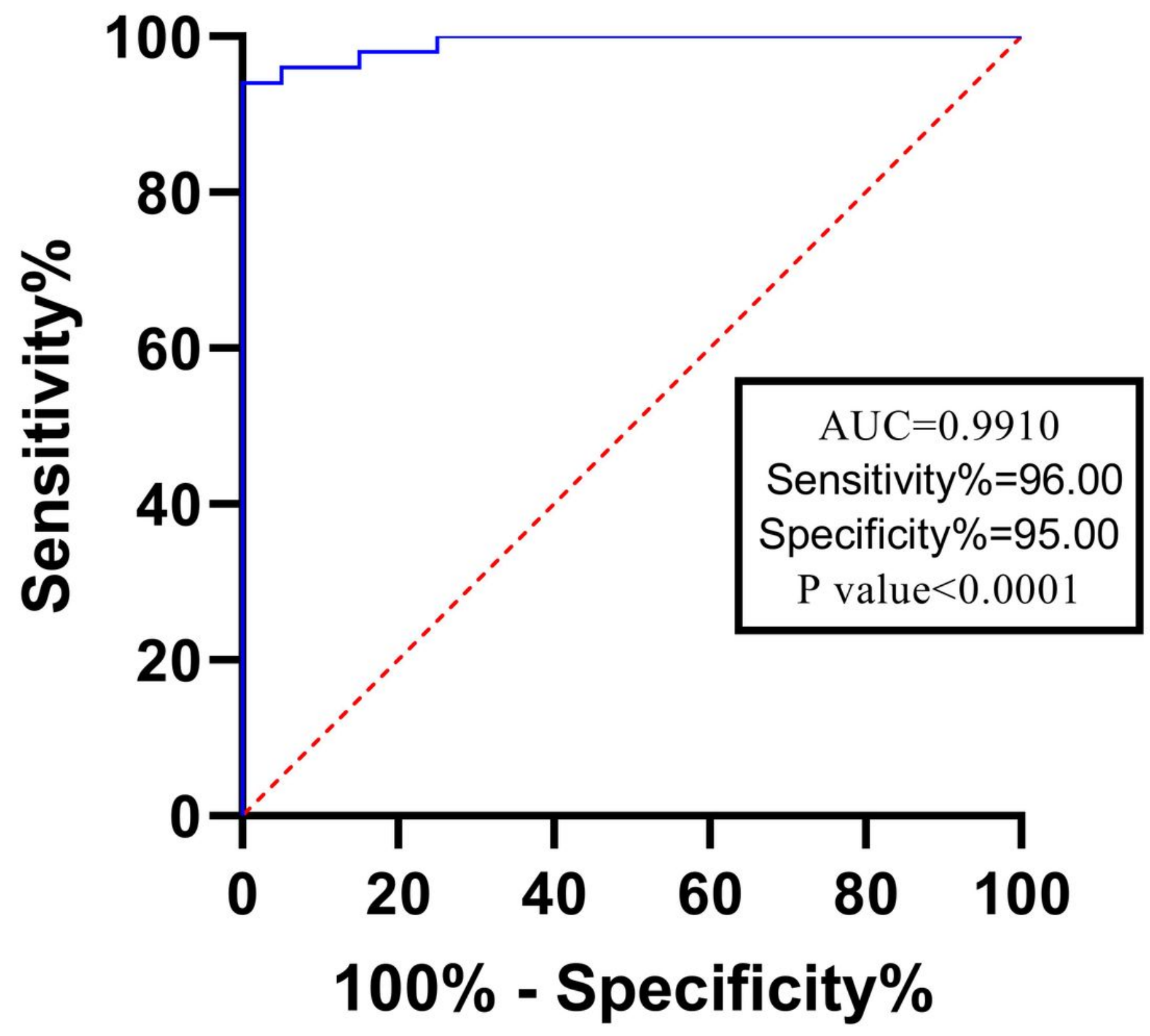

Figure 2

receiver operative characteristic $(\mathrm{ROC})$ curve of relative expression data for miR-27a with a 0.99 area under the curve illustrates a good differential marker for diagnosing systemic sclerosis patients. P-value $=0.0001$, $95 \%$ confidence interval $=0.976$ to 1.000 . 


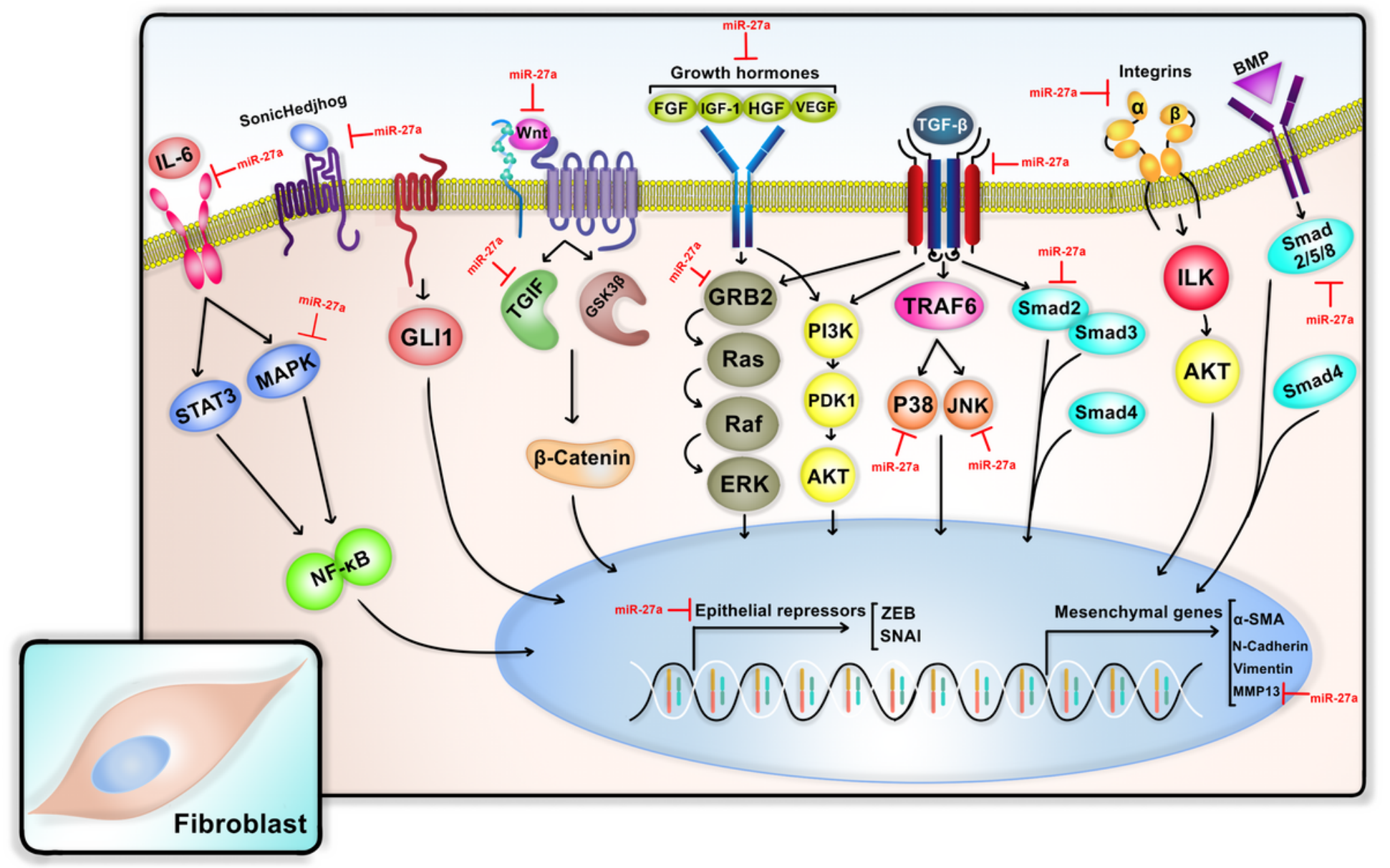

Figure 3

predicted targets of miR-27a in the EMT pathway. miR-27a could interact with and target multiple gene transcripts contributing to the EMT signaling pathway which eventually results in the differentiation of fibroblasts into myofibroblasts; these cells are responsible for the development of fibrosis which is a key feature of SSc. 